\title{
Menschenrechte in der europäischen Rechtspflege ${ }^{1}$
}

\section{- Kritik der Rechtsentwicklung und konstruktive Perspektiven für den Rechtsanwender vor Ort -}

Menschenrechte in der europäischen Rechtspflege ist ein schwieriges, voraussetzungsreiches Thema. Es enthält drei Elemente: Rechtspflege, Europa und Menschenrechte. Jedes dieser drei Elemente wäre eine eigene Abhandlung wert. Das Thema läßt sich in drei Schritten entfalten, an deren Ende ein Ausblick steht, der einen Rahmen für künftige Rechtsentwicklungen in Europa anbietet. Zunächst geht es um das, was sich in Europa derzeit an Rechtspflege erkennen lässt. Man muß sehr genau hingucken. Auf den ersten Blick - auch auf den zweiten und dritten - lassen sich eher weniger Beispiele für die Pflege des Rechts als vielmehr dramatische Belege für dessen Verwahrlosung erkennen (I). Im zweiten Schritt geht es darum, einen maßvollen Optimismus zu verbreiten, indem verschiedene aktuelle Versuche europäischer Rechtspflege aufgezeigt werden. Es wird sich aber erweisen, dass es diesen Versuchen an einem juristischen Kompaß fehlt, der die Richtung anzeigt, welche die europäische Rechtsentwicklung nehmen soll (II). Der dritte Schritt ist der wichtigste: Er entfaltet die Idee der Menschenrechte als absolute, stets verbindliche und unverfügbare Prinzipien des Rechts. Er enthält ein paar wichtige Anwendungsbeispiele, die maßgebend für künftige Rechtsentwicklungen sein können (III).

\section{Europäische Rechtspflege in der Krise}

\section{Affären und ihre Bedeutung}

Die europäische Rechtspflege ist in der Krise. Europäische Rechtspflege meint dabei zum einen den Bezugsrahmen, der sich auf überstaatlicher Ebene um das nationale Recht gelegt hat, enthält also die exekutivischen, legislativen und judikativen Elemente europäischen Rechts, die das staatliche Recht erfassen. Der Begriff enthält zum anderen aber auch die gemeinsamen Merkmale der staatlichen Justizsysteme. Vergleicht man diese wird die Krise der europäischen Rechtspflege staatsübergreifend deutlich. So ist das politische System Frankreichs gerade durch die Justizaffäre von Outreau erschüttert worden. ${ }^{2}$ Nur aufgrund der Zeugenaussage eines Kindes hat ein übereifriger französischer Untersuchungsrichter 14 Einwohner eines kleinen Dorfes in Frankreich des sexuellen Missbrauchs an Kindern verdächtigt, vernommen und Untersuchungshaft angeordnet. Die Beschuldigten werden medial in ihrer bürgerlichen Existenz vernichtet, zwei

1 Vortrag gehalten auf der Jahrestagung der Neuen Richtervereinigung am 16. Juni 2006 in Malente. Der Vortragsstil wurde überwiegend beibehalten.

2 Vgl. zum folgenden Outreau, les étapes d'une catastrophe judiciaire, in: Le Monde vom 7. Dezember 2005, S. 26 f. 
der Beschuldigten nehmen sich während der langen Haftdauer das Leben. Am Ende stellen sich die Beschuldigungen als unwahr heraus. Alle Beschuldigten sind Opfer einer persönlichen, medial aufgeheizten Inszenierung eines sexuellen Gewaltverbrechens, das stets im Fokus politischer Bedrohungsszenarien steht. Sie werden öffentlich, nicht zuletzt durch Anhörungen in der französischen Nationalversammlung rehabilitiert. Diese Anhörungen sind zum Teil erschütternde Dokumente vom Verlust justizieller Kontrolle im strafrechtlichen Ermittlungsverfahren, vom Abgesang der Unschuldsvermutung, von der Sicherheitshysterie, die bedenkenlos jedes Justizgrundrecht $\mathrm{zu}$ opfern bereit ist, wenn es dem trügerischen Schein effektiver Verbrechensbekämpfung dient. ${ }^{3}$ Eine andere fast gleichlautende Affäre - Dutroux - ist im Nachbarland Belgien immer noch nicht verarbeitet. Auch hier bietet sich ein ähnliches Bild: es zeigt justizielle Machtlosigkeit gegenüber machtvoller, dafür aber auch umso unfähigerer Polizeiarbeit. ${ }^{4}$ Ein weiteres Nachbarland sei nicht ausgespart: In Luxemburg geht Ende Februar ein Zellenblock der einzigen Strafvollzugsanstalt des Landes in Flammen auf - Resultat von Überbelegung, zu langer Haftdauer und einem durch Sicherheitsfragen gänzlich überlasteten Strafvollzugssystem. ${ }^{5}$ Auf Deutschland, das heißt auf die noch ungeklärte Beteiligung von Geheimdiensten in Ermittlungsverfahren, auf ungerechtfertigte Rasterfahndung, auf Landespolizeigesetze ohne Gefahrbegriff, auf die schutzlose Auslieferung eigener Staatsangehöriger, auf die Verwahrung von Bürgern, die als Risiko gelten und den populistischen Interessen an flächendeckender Sozialkotrolle, ist gleich zurückzukommen. Die europäische Rechtspflege ist in der Krise - jeder Mitgliedstaat der Europäischen Union trägt mit den rechtsstaatlichen Defiziten seines Kriminaljustizsystems dazu bei.

\section{Beleg der Krise: Europäische Kriminalpolitik}

Aber nicht nur einzelstaatliche Krisen verdichten sich zu einer Krise der europäischen Rechtspflege. Auch Europa selbst, genauer: die Institutionen und die Praxis der Europäischen Union bei der Entwicklung und dem Vollzug europäischen Strafrechts nehmen diese krisenhaften Erscheinungen auf und verdichten sie. Die europäische Kriminalpolitik meint es gut mit der europäischen Rechtspflege und scheitert, ohne es erfahren und wissen zu wollen. Sie erkennt die Krise der europäischen Rechtspflege, freilich die falsche. Sie benennt Rezepte, freilich die ungeeigneten. Sie weist Auswege, die nur noch tiefer in die Problemlagen hineinführen. Genannt seien drei Beispiele:

\section{a) Das Haager Programm der Europäischen Union}

Der Europäische Rat hat das Haager Programm der Europäischen Union am 5. November 2004 gebilligt. ${ }^{6}$ Seine Zielsetzung ist kräftig formuliert. Es soll nicht weniger

3 Vgl. auch Soulez-Larivière, Outreau et puis rien, in: Le Monde vom 7. Dezember 2005, S. 23.

4 Vgl. dazu bereits Matray, Le chagrin des juges, 1997; de Clerck, Les arbres et la forêt, 1997.

5 Vgl. »Le Jeudi«, 02.02.2006, S. 6.

6 Rat der Europäischen Union, Haager Programm zur Stärkung von Freiheit, Sicherheit und Recht in der Europäischen Union, 16054/04; vgl. auch Mitteilung der Kommission an den Rat und das Europäische Parlament - Das Haager Programm : Zehn Prioritäten für die nächsten fünf Jahre (...). KOM(2005), 184 endg. 
als Freiheit, Sicherheit und Recht in der Europäischen Union stärken. Basierend auf der Maxime der gegenseitigen Anerkennung justizieller Entscheidungen verschreibt es sich ganz der Idee, das Strafrecht Europas legitimiere sich dann am besten, wenn es Ängste von Bürgern aufgreift und Rezepte zur Angsttherapie anbietet. So vielfältig die Ängste sind, so heterogen sind auch die Lösungen, die Europas Strafrecht bieten soll. Das europäische Strafrechtsprogramm, das dort formuliert wird, liest sich wie die Hochglanzbroschüre einer Bausparkasse. Sie haben Sorgen? Wir sind für sie da! Sie haben Angst vor Bin Ladin? Wir haben den europäischen Haftbefehl! Sie fürchten sich vor dem Verlust von Haus und Hof? Wir haben ein Strafrecht zum Schutz finanzieller Interessen! Sie wollen keine Hooligans, welche den Fußball-Spaß trüben? Wir haben Europol!

Was das Programm vor allem kennzeichnet, ist administrativer Aktivismus. Es unterbreitet insgesamt 345 Vorschläge, die in den Jahren bis 2009 durch europäischen Rechtsakt umgesetzt werden sollen. ${ }^{7}$ Dabei zählt Kohärenz nicht zu den programmatischen Stärken. Die Maxime gegenseitiger Anerkennung soll umfassend wirksam werden. Man will die gegenseitige Anerkennung der Beweiserhebung ermöglichen, setzt sich europaweit für eine erleichterte Übermittlung personenbezogener Daten ein. Europol und Eurojust sollen ausgebaut werden - bis hin zu einer nachrichtendienstlich gestützten Strafverfolgung. Erst dann, wenn staatenübergreifende Strafverfolgung und Gefahrenabwehr erleichtert wurden, will man sich um Mindestnormen für den Beweismitteltransfer und um prozessuale Mindeststandards bemühen. 2008 immerhin und schon auf Platz 326 des Progamms zu finden - will die Europäische Union ein neues Grünbuch für prozessuale Mindeststandards vorlegen. ${ }^{8}$ Das macht wahrlich Hoffnung: Zum einen ist der erste Versuch eines Rahmenbeschlusses über Verfahrensgarantien gerade auf der Tagung des Europäischen Rates in Wien gescheitert. Nur wenige Mitgliedstaaten sprachen sich für einen mehr als selektiven Prinzipienkatalog aus, der zudem noch auf europäischer Ebene wirksam justiziell kontrolliert werden sollte. Zum anderen wird das Grünbuch vermutlich schmal ausfallen, weil nach vorangegangener Rechtsvernichtung nichts mehr von europäischen Rechtsprinzipien übrig bleiben wird. Das Haager Programm enthält die Betriebsanleitung für eine kraftvolle Exekutive, die den Konflikt zwischen Freiheit und Sicherheit, zwischen Justizförmigkeit und Verfolgungseffizienz stets zum Nachteil des Rechts und zum Vorteil politischer Funktionalität auflöst.

\section{b) Europäische Betrugsermittlungen}

Ein zweites Beispiel bietet das System europäischer Betrugsermittlung, das vor allem durch die Europäische Betrugsbekämpfungsbehörde OLAF geprägt ist. Selten werden die Probleme dieser administrativen Vorermittlungsinstanz öffentlich diskutiert. Einzig der Fall des Sternjournalisten Tillack bot öffentliche Einblicke in die Probleme, die diese europäische Institution bereitet. ${ }^{9}$ Der Journalist hatte kritisch über korruptive

7 Vgl. $\operatorname{KOM}(2005), 184$ endg., Anhang, S. 9 ff.

8 Vgl. aaO., Anhang, S. 21, Punkt 326.

9 Vgl. EuGH Rs. T-193/04, Beschluss vom 15. Oktober 2004 und EuGH Rs. C-521/04, Beschluss vom 19. April 2005. 
Praktiken innerhalb der Europäischen Union berichtet. Nach Ansicht von OLAF-Ermittlern konnte der Bericht nur auf Insiderinformationen beruhen. Tillack wurde verdächtigt, sich diese Informationen seinerseits durch finanzielle Zuwendungen an Kommissionsbeamte beschafft zu haben. Zwei Jahre ermittelte die Behörde gegen den Journalisten und übermittelte das Ergebnis ihrer Ermittlungen an die Staatsanwaltschaft in Brüssel. Rechtsgrundlage für diesen Informationstransfer bildete Art. 10 der OLAF-Verordnung 1073/99. ${ }^{10}$ Man muß wissen, daß es in Belgien zum Zeitpunkt der Weiterleitung von OLAF-Ermittlungsergebnissen kein eigenes Zeugnisverweigerungsrecht für Journalisten, folglich auch keine Beschlagnahmeverbote gab. Die belgische Polizei durchsuchte Wohnung und Büro des Journalisten, beschlagnahmte Unterlagen, Kontoauszüge, Computer, Adreßbücher. Läßt sich die Weitergabe einer administrativen Vorermittlung an nationale Instanzen mit der Folge der Einleitung eines Strafverfahrens und damit verbundener Zwangsmittel europarechtlich verhindern? Der Europäische Gerichtshof verneinte diese Frage. Art. 10 VO 1073/99 sei eine unverbindliche, informelle Regel. Rechtsschutz gegen von OLAF veranlaßte strafrechtliche Ermittlungen könne nur vor den nationalen Gerichten erlangt werden. ${ }^{11}$

Hier verkennt der EuGH die Strafrechtsrelevanz administrativer Betrugsermittlungen völlig. OLAF übermittelt Beweistatsachen, die in ihrer Konsequenz einen Verdacht begründen und damit ein Strafverfahren auslösen können. Freilich bleiben die OLAF-Ermittlungen selbst im Dunkeln und erweisen sich weder durch den Betroffenen, auch nicht durch nationale Gerichte als überprüfbar. Das vage, unverbindliche, informelle Normprogramm der OLAF-Verordnungen hat eine unübersehbare Rechtsschutzlücke in seinem Rücken. Es wird europäisch verfolgt, aber nicht europäisch justizförmig kontrolliert. ${ }^{12}$

\section{c) EuGH und EG-Kompetenzen}

Das letzte Beispiel betrifft noch einmal den Europäischen Gerichtshof und seine neueste Rechtsprechung zu den Kompetenzen der Europäischen Gemeinschaft im Bereich des Strafrechts. In seinem Urteil vom 13. September 2005 hat der Europäische Gerichtshof der Europäischen Kommission die Kompetenz zugesprochen, per Richtlinie umweltstrafrechtliche Regeln der Mitgliedstaaten harmonisieren zu können. ${ }^{13}$ Das Gericht hat sich damit gegen eine Regelungskompetenz im Rahmen der Dritten Säule, also im Bereich der völkervertraglichen Zusammenarbeit der Mitgliedstaaten gewandt. Zwar mag das Urteil zunächst nur auf den spezifisch ausgewiesenen Bereich des europäischen Umweltrechts zugeschnitten sein, aber schon jetzt ist absehbar, dass die Europäische Kommission daraus eine allgemeine strafrechtliche Rechtsetzungskompetenz ableitet. Schon jetzt sind die Demokratiedefizite in der Entwicklung europäischen Strafrechts schwerwiegend. Nun droht die legalisierte Vorherrschaft der Exe-

10 ABl. L 136/1 vom 31.5.1999.

11 EuGH 2004, 43. Erwägungsgrund; EuGH 2005, 31., 32. und 38. Erwägungsgrund.

12 Vgl. dazu Verf., wistra 2005, $401 \mathrm{ff}$.

13 EuGH, Rs. C-176/03 (Europäische Kommission vs. Europäischer Rat), Urteil vom 13. September 2005. 
kutive im europäischen Strafrecht. Diese Vorherrschaft überlastet das Strafrecht, degradiert es zu einem überspannten administrativen Sanktionenrecht ohne rechtsstaatliche Kontur, jenseits demokratischer Legitimation. ${ }^{14}$

\section{Die Zerstörung juristischer Professionalität}

Man kann verschiedene Formeln finden, um diese Krise europäischer Rechtspflege und ihre Symptome zusammenzufassen. Es geht um Rechtserosionen, ja um Vernichtungen, um einen dramatischen Funktionswandel des Strafrechts, der über bloße Prävention hinausweist. Davon abgesehen wird all das zerstört, was man Studierenden und Referendaren lehrt, was in der Praxis der Gerichte, der Staatsanwaltschaften und der Verteidigung gepflegt wird: solide juristische Professionalität, also das sachgerechte Subsumieren eines Sachverhalts unter einen Rechtssatz, transparentes, kohärentes gar kritisches Argumentieren, die Orientierung an den Folgen einer juristischen Entscheidung, die Wahrung von Frist und Form.

Im Strafrecht kann man sich auf diese Merkmale eines professionellen Umgangs mit Regeln nicht mehr verlassen. ${ }^{15}$ Regel und Kommentierung der Regel allein bieten keine solide Grundlage mehr für eine überzeugende, allgemeinverbindliche juristische Argumentation. Die Krise ist gerade eine Krise, weil das juristische Handwerkszeug nicht mehr funktionsfähig ist. Woran liegt das? Offenbar scheitert der tradierte technische Zugang zu rechtlichen Problemlagen aus drei Gründen, die zugleich drei beklagenswerte Verluste darstellen - einen Verlust an Verbindlichkeit, einen Verlust an Vertrauen, einen Verlust an Staatlichkeit.

So haben zum einen die Regeln, die der Gesetzgeber aufstellt, an normativer Verbindlichkeit, auch an Verläßlichkeit verloren. Der Gesetzgebungsaktionismus folgt dem Prinzip des »try and error «, die Regel wird erlassen, um sie gleich nachzubessern, die Geltungsdauer der Regel verkürzt sich. Teils setzt der Gesetzgeber auf Regeln, die auf Zeit, gleichsam experimentell erlassen werden. Rechtseinschnitte, etwa die Rasterfahndung oder die Kronzeugenregelung, sollten auch durch zeitlich begrenzte Geltung erträglich und damit zustimmungsfähig gemacht werden. Mit einem französischen Sprichwort läßt sich diese Experimentalgesetzgebung einordnen: »Il n'y a que des provisoires qui durent » : Nur das Vorläufige ist wirklich von Dauer. Die Gesetzesqualität, die daraus erwächst, ist schlecht. Vorschriften wie die Geldwäsche, der Insiderhandel, der Subventionsbetrug und viele andere Delikte des Nebenstrafrechts sind kaum handhabbar. Es bleibt der Justiz nur die Flucht in Opportunität, Diversion und Informalität, um der Flut an Experimentalgesetzgebung Herr zu werden. ${ }^{16}$

Zum anderen hat die normative Verläßlichkeit des demokratischen Gesetzgebers selbst Schaden genommen. Mehr denn je ist Mißtrauen unabhängiger Rechtspflege gegenüber den Pflegeprodukten des Gesetzgebers angebracht. Kaum jemals zuvor in der Verfassungsgeschichte der Bundesrepublik hat das Bundesverfassungsgericht, derart

14 Vgl. auch Verf., wistra 2006, $121 \mathrm{ff}$.

15 Vgl. dazu bereits Naucke, Versuch über den aktuellen Stil des Rechts, KritV 1986, $201 \mathrm{ff}$.

16 Vgl. schon P.-A. Albrecht, Exekutivisches Recht, in: ders. (Hrsg.), Informalisierung des Rechts, 1990, S. 1 ff. 
umfangreich und in derart kurzer Zeitabfolge, (straf-)gesetzgeberische Fehlentscheidungen teils korrigieren, teils gänzlich aufheben müssen. Die Entscheidungen zum europäischen Haftbefehl ${ }^{17}$, zur akustischen Wohnraumüberwachung ${ }^{18}$, zur Sicherungsverwahrung $^{19}$, zum Luftsicherheitsgesetz ${ }^{20}$, nun zur Rasterfahndung ${ }^{21}$ dokumentieren, wie erschreckend weit der Gesetzgeber von verfassungsrechtlich geschützten Rechtsprinzipien teilweise entfernt ist. Eine populistische Kriminalpolitik versucht stets ihre Handlungsspielräume zu erweitern - die Verformung der Verfassung ist dabei einkalkuliert.

Schließlich verdeutlichen die europäischen Krisenmerkmale, daß kein Bereich praktischer Rechtsanwendung von Einflüssen überstaatlichen Rechts unbeeindruckt bleibt. Selbst der schnöde Zugang einer Willenserklärung kann Gegenstand komplexer EuGH-Rechtsprechung, europäischer Rechtsakte und sogar auch eine Menschenrechtsfrage sein ${ }^{22}$.

Was folgt zunächst aus dieser Diagnose einer zerstörten Professionalität des Rechts? Wenn die Technik der positiven Regel versagt, muß sich die Orientierung im Recht, insbesondere innerhalb der dritten Gewalt, aber auch genereller im wissenschaftlichen Umgang mit dem Recht, verändern. Am Ende einer zerstörten Professionalität, - so eine Formulierung Ronald Dworkins - wird klar, daß rechtswissenschaftliche Fragen im Kern Prinzipienfragen $\operatorname{sind}^{23}$, nicht Fragen des flüchtigen, seiner festen Positivität beraubten positiven Rechts. Wer auf einem bloß konventionellen positivistischen $\mathrm{Zu}$ griff auf das Recht besteht, trägt dessen Fundamente mit zu Grabe. Angesichts der beschriebenen Verluste an juristischer Professionalität kommt es entscheidend darauf an, die Legitimität von Recht zu hinterfragen, Farbe zu bekennen und den menschenrechtlichen Gehalt juristischen Entscheidens aufzuarbeiten, offenzulegen und im besten Fall durchzusetzen.

\section{Versuche europäischer Rechtspflege}

Auf europäischer Ebene gibt es wohlmeinende Versuche, der Krise europäischer Rechtspflege Herr zu werden. So hat eine international besetzte Arbeitsgruppe von Strafrechtsprofessoren unter der Leitung des Münchner Strafrechtslehrers Schünemann vor kurzem ein Gesamtkonzept für eine europäische Strafrechtspflege vorgelegt. ${ }^{24}$ Das Konzept ist wichtig. Es beharrt auf einem prinzipienorientierten Verständnis der gegenseitigen Anerkennung im Strafrecht, will am Grundsatz der

17 BVerfG, 2BvR 2236/04 vom 18.7.2005.

18 BVerfG, 1 BvR 2378/98 vom 3.3.2004.

19 BVerfG, 2BvR 834/02 vom 10.2.2004.

20 BVerfG, 1BvR 357/05 vom 15.2.2006.

21 BVerfG, 1 BvR 518/02 vom 4.4.2006.

22 Vgl. etwa $H e \beta$, NJW 2001, 15 ff.

23 Vgl. Dworkin, Bürgerrechte ernstgenommen, 1990, S. 30.

24 Das »Gesamtkonzept für europäische Strafrechtspflege « wurde am 26. und 27. Mai 2006 in Thessaloniki vorgestellt und diskutiert. Vgl. dazu auch F.-C. Schroeder, FAZ vom 1. August 2006. 
beiderseitigen Strafbarkeit festhalten und pocht - zu Recht - auf eine wirksame Etablierung von Rechten der Strafverteidigung auf einer gesamteuropäischen Ebene. Das materielle Strafrecht soll auf den Schutz von besonders bedeutsamen individuellen, aber auch von kollektiven Rechtsgütern beschränkt sein. ${ }^{25}$ Die Diskussion um das Konzept hat zwei Probleme offenbart - ein inhaltliches und ein formelles.

Das inhaltliche Problem besteht im Verständnis von Strafverteidigung. Strafverteidigung in Europa soll durch eine Institution namens »Eurodefensor « gesichert werden. In »Eurodefensor « sollen Beamte der EU für die Pflege der Strafverteidigung zuständig sein. In Anlehnung und Anpassung an das Gefüge europäischer Institutionen, ließe sich selbst die Organisation der Strafverteidigung exekutivisch ausrichten. So wichtig es ist, die Strafverteidigung zum vorrangigen Gegenstand europäischer Kriminalpolitik zu machen, so darf man die wirksame Koordinierung von Strafverteidigern in Europa keinesfalls EU-Beamten überlassen. Das hieße nun wahrlich den Bock zum Gärtner zu machen.

Das formelle Problem betrifft die kommunikative Vermittlung in andere Rechtskulturen. Der Gedanke kollektiver Rechtsgüter und die differenzierten Debatten, wann deren strafrechtlicher Schutz legitim sein soll, entstammen vor allem der deutschen Rechtstradition. Ein englischer oder französischer Strafrechtler kann die traditionsbedingte, inhaltsspezifische Differenzierung dieser Debatte nicht unmittelbar nachvollziehen. Hier greifen andere - ebenso differenzierte - dogmatische Traditionen. So fehlt es bereits an einer tragfähigen Übersetzung des Begriffs vom kollektiven Rechtsgut ins englische oder französische. Rechtsgut meint in diesen Sprachen immer »interest « oder »intérêt « und damit - ganz undifferenziert - alles und nichts. Eine Begrenzungskonzeption läßt sich damit rechtskulturübergreifend nicht vermitteln.

Auch der gerade gescholtene EuGH versucht sich an der europäischen Rechtspflege. In seinem Urteil vom 30. Mai 2006 hat er den Beschluß des Europäischen Rates über das Flugdatenabkommen mit den USA für nichtig erklärt. ${ }^{26}$ Freilich leider nicht aus naheliegenden Gründen des Datenschutzes, sondern weil die Europäische Union keine Kompetenz zum Abschluß eines solchen Abkommens habe.

So fehlt es beiden Versuchen gerade an Farbe, weil sie eine Konzeption von Menschenrechten als Fundus gemeinsamer europäischer Rechtstradition und als Grundlage jeglicher europäischer Rechtsentwicklung überwiegend vermissen lassen.

III. Menschenrechte - Konzept europäischen Strafrechts und seine Implementation

Die Frage lautet also: Wie begegnet man der Krise europäischer Rechtspflege? Und die Antwort muß sein: Durch das Festhalten, das Neuentwickeln und das Durchsetzen eines präzisen Begriffs des Rechts, der die Freiheit als Grundprinzip wiederentdeckt und der dieses Grundprinzip in individuelle Rechte umsetzt, die jenseits des Staates zu aller Zeit, an jedem Ort der Welt gelten. Wie rechtfertigt man solch große Worte?

25 Vgl. auch schon Schünemann (Hrsg.), Alternativentwurf europäische Strafverfolgung, 2004.

26 Europäischer Gerichtshof, Rechtssachen C-317/04 und C-318/04, Urteil vom 30. Mai 2006. 


\section{Freiheit im Staat und über den Staat hinaus}

Ein Kernsatz der politischen Philosophie der Aufklärung lautet, daß man das Wagnis eingehen solle, von seinem eigenen Verstand Gebrauch zu machen ${ }^{27}$, sich aus der verführerischen Einfachheit gedanklicher Anpassung an staatliche, religiöse oder gesellschaftliche Gepflogenheiten zu befreien. Der Satz stammt von Kant, der darauf pocht, daß Menschen als Staatsbürger stark genug sind, ihr eigenes Schicksal in die Hand zu nehmen. Sie sind aber auch andererseits schwach genug, um vor fremder Stärke - der von Staaten, Religionen oder gesellschaftlichen Mehrheiten - beschützt werden zu müssen. Freiheit birgt Risiken, die dann eintreten, wenn die Freiheit des anderen nicht anerkannt und verletzt wird. Ohne Freiheit aber droht die entmenschlichte Realität einer Gemeinschaft tumber Individuen, die durch jede Heilslehre verführbar und jeder auch noch so irrationalen Neigung nachzugeben bereit sind. Freiheit macht einen wichtigen Teil der menschlichen Würde aus. Ihr Verlust und Nichtgebrauch ließe unser Dasein verkümmern. Das gilt für den Staat und darüber hinaus.

Freilich macht die Freiheit - Kant berücksichtigt das im »Ewigen Frieden« - nicht an Staatsgrenzen Halt. In der Form des Handelsgeistes sprengt er staatliche Grenzen und fordert den nur staatlichen Schutz der Freiheit neu heraus - wenngleich nicht in dem Sinne, daß man sich vor einem plötzlich auftretenden Zuviel an Freiheit in Sicherheit bringen müßte. Im Gegenteil: Auch über staatliche Grenzen hinweg sollen Menschen von ihrem eigenen Verstand Gebrauch machen können. Kant nennt diese staatenübergreifend agierenden Individuen »Weltbürger« und schreibt ihnen ein Weltbürgerrecht zu, das sich als Freiheit von jeglichem staatlichen Zwang interpretieren läßt. Die Garantie dieses Rechts bildet zugleich den kritischen Maßstab für das staatliche Handeln selbst. ${ }^{28}$ Aus der Freiheit, die das Weltbürgerrecht auch jenseits des Staates enthält, leiten sich - ex negativo - individuelle Abwehrrechte gegen den Staat selbst, aber auch gegen Staatengemeinschaften, überhaupt gegen jede Zusammenballung politischer Macht im Staat und über den Staat hinaus ab. ${ }^{29}$ Wichtig ist, daß es sich nicht um irgendwelche Rechte handelt, sondern um Rechte von besonderer Qualität, die den Menschen als Rechtsperson erst hervorbringen. Abwehrrechte bedeuten Grundlegendes. Sie umfassen das Recht auf Leben und körperliche Unversehrtheit, auf Eigentum, auf Meinungs- und Willensfreiheit, schließlich auch die gerechte Chance, diese Rechte wirksam geltend machen zu können - das Recht auf ein faires Verfahren also.

\section{Recht und Interesse}

Wem es übertrieben oder unverständlich scheint, die deutschen Klassiker zu bemühen, um die Bedeutung der Menschenrechte als staatenloses Recht gegen den Staat zu beto-

27 Kant, Beantwortung der Frage: Was ist Aufklärung? (AA VIII, S. 35).

28 Vgl. Verf., Europäische Strafgesetzlichkeit, 2003, S. 88 ff; ähnlich jetzt auch Gierhake, Begründung des Völkerstrafrechts auf der Grundlage der Kantischen Rechtslehre, 2005, insbesondere S. $239 \mathrm{ff}$.

29 Vgl. näher Verf., Europäische Strafgesetzlichkeit, 2003, S. 73 ff. 
nen, mag sich auf andere Quellen stützen. Die Begründung der Menschenrechte liegt auf einer Erkenntnislinie, die in verallgemeinerbarer Weise Rechte von bloßen Interessen zu unterscheiden weiß. Amerikanische Rechtsphilosophen wie Dworkin oder Rawls weisen darauf hin, daß die Menschenrechte nicht nur jegliche staatliche Souveränität einschränken, sondern auch einem beliebigen Pluralismus Grenzen setzen. ${ }^{30}$ Nur weniges ist wirklich von zeitloser Dauer - Menschenrechte zählen dazu. Daneben gibt es Interessen, die von Mehrheiten artikuliert und politisch kraftvoll durchgesetzt werden, etwa Interessen an stabilen Sozialsystemen, an wirtschaftlicher Prosperität, an der Verringerung persönlicher Lebensrisiken. Interessen können rationale gesamtgesellschaftliche Zielsetzungen sein oder abwegige Reflexe der Freizeitgesellschaft. Mitunter ist ein Unterschied in den aktuellen kriminalpolitischen Debatten nicht zu erkennen. Unlängst konnte man in der Begründung eines Gesetzesentwurfs zur Neufassung des Sachbeschädigungstatbestandes lesen, Graffiti verletzten die »Ästhetik des Lebensgefühls « und seien daher in jedem Falle strafbar ${ }^{31}$. Das ist eine ernstgemeinte Begründung, den guten Geschmack zum Rechtsgut zu erheben. Heute kriminalisiert man noch Graffiti-Sprayer, morgen vielleicht Tennissockenträger oder englische Köche.

Auch der Staat hat Interessen, etwa an seinem Fortbestand an äußerer und innerer Sicherheit. Dworkin argumentiert indes, daß das Sicherheitsinteresse des Staates kein eigenes Recht darstellt, sondern vielmehr die Summe persönlicher Vorlieben einer Vielzahl von Personen. ${ }^{32}$ Es gibt demnach kein Grundrecht auf Sicherheit, nur die individuelle Neigung der Mehrheit zu einem sorgen- und angstfreien Leben. Diese Neigungen können sich so oder anders artikulieren, es fehlt ihnen an qualitativer Verallgemeinerbarkeit, die das Interesse zum Recht und das Recht zum Menschenrecht machen. Aus Bausparkassenbroschüren wird keine Verfassung.

\section{Vernichtung der Freiheit als historische Erfahrung}

Schließlich - auch diese Erkenntnislinie ist wichtig - bestätigt sich die politische Freiheitsphilosophie durch die europäische Geschichte, die von der Vernichtung der Freiheit und den Folgen dieser Vernichtung handelt. Weil das Ausmaß staatlicher Freiheitsvernichtung in der europäischen Geschichte - insbesondere im Nationalsozialismus - schmerzlich erfahrbar war, entfalteten die Allgemeine Erklärung der Menschenrechte und die Europäische Konvention zum Schutze der Menschenrechte und Grundfreiheiten ihre praktische Wirkung im kodifizierten Völkerrecht. ${ }^{33}$ Damit sind wir zum Schluß bei der praktischen Durchsetzung von Menschenrechten, die sich an drei Beispielen aus der Rechtsprechung des Europäischen Gerichtshofs für Menschenrechte verdeutlichen lassen.

30 Vgl. einerseits Dworkin, Bürgerrechte ernstgenommen, 1990, S. 144 ff. und andererseits Rawls, Das Recht der Völker, 2002, S.29 f.

31 Vgl. den Gesetzesentwurf des Bundesrates für ein Graffiti-Bekämpfungsgesetz aus dem Jahr 1999, BT-Drucks. 14/872.

32 Vgl. Dworkin, aaO., u.a. S. 54 ff. und S. $429 \mathrm{ff}$.

33 Vgl. auch die Argumentation von Rawls, Das Recht der Völker, 2002, S. 22 ff. 


\section{Menschenrechte in der justiziellen Praxis}

a) Geheime Ermittlungen

Die ersten beiden Beispiele betreffen die Problematik geheimer Ermittlungsmethoden im Strafverfahren. Im Fall Edwards und Lewis gegen das Vereinigte Königreich ${ }^{34}$ waren die Kläger von einem polizeilichen Lockspitzel in einen Drogentransport verwickelt worden. Die Identität des Lockspitzels wurde im Verfahren geheimgehalten, aber auch die Beweise, auf die sich die Verurteilung maßgeblich stützte - insbesondere Notizen und Protokolle des geheimen Ermittlers - haben weder der Beschuldigte noch sein Verteidiger je zu Gesicht bekommen. So konnten weder die Beweistatsachen kritisch gewürdigt, noch Fragen an den Hauptbelastungszeugen gestellt werden - eine Situation, wie sie auch im deutschen Strafprozeß nur allzuoft vorkommt. In seinem Urteil vom 27. Oktober 2004 hat der Europäische Gerichtshof für Menschenrechte der Klage stattgegeben und eine Verletzung des Fair-Trial-Prinzips aus Art. 6 EMRK festgestellt. In seiner Begründung verankert der EuGHMR einige wichtige Pflöcke bei der praktischen Anwendung von Art. 6 EMRK. Ein faires Verfahren verlange nach Waffengleichheit und wirksamer Verteidigung. Das setzt voraus, daß Beschuldigter und sein Verteidiger über alle wichtigen, be- und entlastenden Beweise verfügen können müssen. ${ }^{35}$ Das öffentliche Interesse der Kriminalitätsbekämpfung sei durch die Rechte aus Art. 6 EMRK begrenzt. Ein solches Interesse könne den Gebrauch von Beweismitteln, die durch geheime Ermittlungsmethoden erlangt wurden, grundsätzlich nicht rechtfertigen. Nur absolut notwendige Einschränkungen der Verteidigung seien zulässig, aber auch nur dann, wenn es sich um besonders wichtige öffentliche Interessen handelt oder um schützenswerte Rechte Dritter. Selbst wenn aus einem solchen Grund, der Verteidigung Beweismittel während des Ermittlungsverfahrens vorenthalten wurden, muß dies durch das Verfahren vor den Gerichten hinreichend ausgeglichen werden. ${ }^{36}$

Im Fall Allan gegen das Vereinigte Königreich ${ }^{37}$ wird diese Linie ebenfalls deutlich sichtbar. Waffengleichheit und wirksame Verteidigung werden durch das Nemo-tenetur-Prinzip ergänzt. Alle drei Prinzipien sind Ausdruck des Menschenrechts auf ein faires Verfahren. ${ }^{38}$ Im Fall ging es um ein Geständnis, das der Kläger gegenüber einem Mithäftling ablegte, den die Polizei in die Gefängniszelle des Klägers eingeschleust hatte. Das Schweigerecht des Beschuldigten schütze ihn vor Zwang und Rechtsmißbrauch durch staatliche Autoritäten. Der Beschuldigte müsse - so der EuGHMR - eine echte Wahlfreiheit haben zwischen Schweigen und Einlassung. Täuscht der Staat eine solche Wahlfreiheit nur vor, so liegt darin eine Verletzung des Menschenrechts auf ein faires Verfahren, insbesondere wenn der Beschuldigte zuvor in offizieller Vernehmung ausdrücklich erklärt hat, von seinem Schweigerecht Gebrauch machen zu wollen. ${ }^{39}$

34 Vgl. EuGHMR Case $\mathrm{N}^{\circ} 39647 / 98$ und $\mathrm{N}^{\circ}$ 40461/98, Urteil vom 27. Oktober 2004.

35 EuGHMR, aaO., S. 14, 5. Absatz (Online-Version).

36 EuGHMR, aaO., 6. Absatz (Online-Version).

37 EuGHMR, Case N ${ }^{\circ}$ 48539/99, Urteil vom 5. November 2002.

38 EuGHMR, aaO., Rz. 44.

39 EuGHMR, aaO., Rz. 50. 
b) Menschenrechte und Terrorismus

Das letzte Beispiel betrifft das problembeladene Verhältnis von Menschenrechten und Terrorismus. In der Rechtsprechung des Europäischen Gerichtshofs für Menschenrechte findet sich ein prominenter Fall, der dieses Verhältnis zum Gegenstand hat: der Fall Öcalan. ${ }^{40}$ Die ersten Vernehmungen Öcalans und das Verfahren, das teilweise vor einem mit Militärrichtern besetzten Gericht stattfand, mißachteten fast sämtliche Elemente eines fairen Verfahrens, wie es der Europäische Gerichtshof für Menschenrechte in Anwendung von Art. 6 EMRK versteht. Gleich aus mehreren Gründen sah das Gericht das Recht auf ein faires Verfahren als verletzt $a^{41}$ :

- Kein anwaltlicher Beistand in der Untersuchungshaft

- Keine freie Kommunikation mit der Verteidigung

- Fehlendes Akteneinsichtsrecht selbst im fortgeschrittenen Prozeßstadium

- Unangemessene Besuchs- und Kontaktsperre zum Nachteil der Verteidigung und vor allem

- Das Fehlen eines unabhängigen Gerichts.

Gerade das Urteil im Fall Öcalan macht deutlich, daß die Menschenrechte überstaatlich gelten, mögen die Interessen des Staates an Sicherheit noch so massiv vorgetragen sein. Die Rechtsprechung im Fall Öcalan unterstreicht die Linie einer unabhängigen europäischen Justiz. Ohne Wenn und Aber, so könnte man die Botschaften der Urteile interpretieren, gilt es, unverbrüchlich an dem Recht eines jeden einzelnen auf die Achtung seiner Würde festzuhalten. Es gibt, so zeigen die Fälle, politische Mächte, die im Angesicht immer neuer Bedrohungs- und Horrorszenarien die Koordinaten des Rechtsstaats zugunsten staatlicher Sicherheitspolitik verschieben wollen. Erst die Achtung der Menschenrechte aber wahrt die Balance zwischen Freiheit und Sicherheit, weil aus der Freiheit des einzelnen die Sicherheit für alle erst erwächst - nicht umgekehrt.

Die drei gezeigten Beispiele sind mutig. Sie verdeutlichen, dass - solange es couragierte Richter gibt - Freiheit und Menschenrechte einer leviathanischen Konzeption von staatlicher Sicherheit nicht geopfert werden. In den Fällen Edwards, Allan und Öcalan macht der Europäsiche Gerichtshof für Menschenrechte aber noch mehr: Er bewahrt durch das Aufdecken von Prinzipienproblemen unabhängige Juristen vor der Zertrümmerung der Regeln professionellen Rechtsdenkens. Mit einem etwas aus der Mode gekommenen Wort läßt sich dies näher beschreiben: Er wahrt die Ehre einer unabhängigen Justiz, und das, was Juristen sich und ihrem Gewissen schuldig sind: das Recht und seine Prinzipien gegen die Zumutungen der Politik zu verteidigen ${ }^{42}$. Man kann im juristischen Alltag, in Gerichtssälen und Amtsstuben, auch in Seminaren und Hörsälen nicht mehr tun als das. Das mag nicht viel sein, ist aber mehr als genug und es bleibt genug zu tun.

40 Öcalan vs. Türkei, Case $N^{\circ}$ 46221/99, Urteil der Großen Kammer vom 12. Mai 2005. Vgl. zum vorangegangenen Urteil vom 12.03.2003 die Besprechung von Kühne, JZ 2003, 670 ff.

41 EuGHMR, aaO., Rz. 133 ff. u. Rz. 148.

42 Vgl. auch »Unsere Ehre - Ronald Dworkin kritisiert die Aufweichung des Rechts«, in: FAZ vom 2. Mai 2006, S. 46. 